\title{
On Locally-Balanced 2-Partitions of Complete Multipartite Graphs
}

\author{
Aram H. Gharibyan ${ }^{\dagger}$ and Petros A. Petrosyan ${ }^{\dagger \dagger}$ \\ ${ }^{\dagger}$ Department of Informatics and Applied Mathematics, YSU, \\ $\ddagger$ Institute for Informatics and Automation Problems of NAS RA \\ e-mail: aramgharibyan@gmail.com, petros_petrosyan@ysu.am, pet_petros@ipia.sci.am
}

\begin{abstract}
A 2-partition of a graph $G$ is a function $f: V(G) \rightarrow\{$ White, Black $\}$. A 2-partition $f$ of a graph $G$ is locally-balanced with an open neighborhood if for every $v \in V(G)$,$$
\|\left\{u \in N_{G}(v): f(u)=\text { White }\right\}|-|\left\{u \in N_{G}(v): f(u)=\text { Black }\right\} \| \leq 1,
$$

where $N_{G}(v)=\{u \in V(G): u v \in E(G)\}$. A 2-partition $f^{\prime}$ of a graph $G$ is locallybalanced with a closed neighborhood if for every $v \in V(G)$,

$$
\|\left\{u \in N_{G}[v]: f^{\prime}(u)=\text { White }\right\}|-|\left\{u \in N_{G}[v]: f^{\prime}(u)=\text { Black }\right\} \| \leq 1,
$$

where $N_{G}[v]=N_{G}(v) \cup\{v\}$. In this paper we give necessary and sufficient conditions for the existence of locally-balanced 2-partitions of complete multipartite graphs.

Keywords: 2-partition, Locally-balanced 2-partition, Equitable coloring, Complete multipartite graph.
\end{abstract}

\section{Introduction}

All graphs considered in this work are finite, undirected, and have no loops or multiple edges. Let $V(G)$ and $E(G)$ denote the sets of vertices and edges of a graph $G$, respectively. The set of neighbors of a vertex $v$ in $G$ is denoted by $N_{G}(v)$. Let $N_{G}[v]=N_{G}(v) \cup\{v\}$. A graph $G$ is called a complete $n$-partite $(n \geq 2)$ graph if its vertices can be partitioned into $n$ nonempty independent sets $X_{1}, \ldots, X_{n}$ such that each vertex in $X_{i}$ is adjacent to all the other vertices in $X_{j}$ for $1 \leq i<j \leq n$. Let $K_{r_{1}, r_{2}, \ldots, r_{n}}$ denote a complete $n$-partite graph with independent sets $X_{1}, X_{2}, \ldots, X_{n}$ of sizes $r_{1}, r_{2}, \ldots, r_{n}$. The terms and concepts that we do not define can be found in $[8,15]$.

A 2-partition of a graph $G$ is a function $f: V(G) \rightarrow\{$ White, Black $\}$. A 2-partition $f$ of a graph $G$ is locally-balanced with an open neighborhood if for every $v \in V(G)$,

$$
\|\left\{u \in N_{G}(v): f(u)=\text { White }\right\}|-|\left\{u \in N_{G}(v): f(u)=\text { Black }\right\} \| \leq 1 .
$$

A 2-partition $f^{\prime}$ of a graph $G$ is locally-balanced with a closed neighborhood if for every $v \in V(G)$,

$$
\|\left\{u \in N_{G}[v]: f^{\prime}(u)=\text { White }\right\}|-|\left\{u \in N_{G}[v]: f^{\prime}(u)=\text { Black }\right\} \| \leq 1 .
$$


The concept of locally-balanced 2-partition of graphs was introduced by Balikyan and Kamalian [12] in 2005, and it can be considered as a special case of equitable colorings of hypergraphs [1]. In [1], Berge obtained some sufficient conditions for the existence of equitable colorings of hypergraphs. In $[7,9,10,14]$, the authors considered the problems of the existence and construction of proper vertex-coloring of a graph for which the number of vertices in any two color classes differ by at most one. In [11], 2-vertex-colorings of graphs were considered for which each vertex is adjacent to the same number of vertices of every color. In particular, in [11], it was proved that the problem of the existence of such a coloring is $N P$-complete even for the $(2 p, 2 q)$-biregular $(p, q \geq 2)$ bipartite graphs. In [12], Balikyan and Kamalian proved that the problem of existence of locally-balanced 2-partition with an open neighborhood of bipartite graphs with maximum degree 3 is NP-complete. Later, they also proved [13] the similar result for locally-balanced 2-partitions with a closed neighborhood. In $[2,3,4]$, the necessary and sufficient conditions for the existence of locallybalanced 2-partitions of trees were obtained. In [6], the authors obtained some necessary conditions for the existence of locally-balanced 2-partitions of Eulerian graphs. In particular, they proved some results on the existence of locally-balanced 2-partitions of rook's graphs and cycle powers.

In the present paper we give necessary and sufficient conditions for the existence of locally-balanced 2-partitions of complete multipartite graphs. A preliminary version of this paper was presented at the 6th Polish Combinatorial Conference, Bedlewo, Poland, 2016 [5].

\section{Main Results}

Before we formulate and prove our results, we introduce some terminology and notation. For any 2-partition $\varphi$ of a graph $G$, we define $\bar{\varphi}$ as follows: for any $v \in V(G)$, let

$$
\bar{\varphi}(v)= \begin{cases}\text { Black, } & \text { if } \varphi(v)=\text { White } \\ \text { White }, & \text { if } \varphi(v)=\text { Black }\end{cases}
$$

If $\varphi$ is a 2-partition of a graph $G$ and $v \in V(G)$, then define \#(v) and \#[v] as follows:

$$
\begin{gathered}
\#(v)=\mid\left\{u \in N_{G}(v): \varphi(u)=\text { White }\right\}|-|\left\{u \in N_{G}(v): \varphi(u)=\text { Black }\right\} \mid, \\
\#[v]=\mid\left\{u \in N_{G}[v]: \varphi(u)=\text { White }\right\}|-|\left\{u \in N_{G}[v]: \varphi(u)=\text { Black }\right\} \mid .
\end{gathered}
$$

Clearly, $\varphi$ is a locally-balanced 2-partition with an open neighborhood (with a closed neighborhood) if for every $v \in V(G),|\#(v)| \leq 1(|\#[v]| \leq 1)$.

If $G$ is a complete $n$-partite graph and $X$ is a part of $G$, then $X$ is called an odd (even) part if $|X|$ is odd $\left(|X|\right.$ is even). If $G$ is a complete $n$-partite graph, then by $m_{1}, m_{2}$, and $m_{\geq 3}$ we denote the number of parts of $G$ with one vertex, two vertices and at least three vertices, respectively. If $\varphi$ is a 2-partition of a complete $n$-partite graph $G$ and $X$ is a part of $G$, then by $W_{X}\left(B_{X}\right)$ we denote the number of White (Black) vertices of the part $X$. If $\varphi$ is a 2-partition of a complete $n$-partite graph $G$, then by $W(B)$ we denote the number of White (Black) vertices in $G$.

We begin with the following simple lemma.

Lemma 1. If $\varphi$ is a locally-balanced 2-partition of $G$, then $\bar{\varphi}$ is also a locally-balanced 2partition of $G$.

Lemma 2. If $K_{r_{1}, r_{2} \ldots, r_{n}}$ has a locally-balanced 2-partition with an open neighborhood, then for any part $X,\left|W_{X}-B_{X}\right| \leq 1$. 
Proof. Let $\varphi$ be a a locally-balanced 2-partition with an open neighborhood of $K_{r_{1}, r_{2} \ldots, r_{n}}$. Suppose, to the contrary, that there exists a part $X^{\prime}$ such that either $W_{X^{\prime}}-B_{X^{\prime}} \geq 2$ or $B_{X^{\prime}}-W_{X^{\prime}} \geq 2$. By Lemma 1., we can assume that there exists a part $X^{\prime}$ such that

$$
W_{X^{\prime}}-B_{X^{\prime}} \geq 2
$$

It is easy to see that for any $v \in X$,

$$
\#(v)=W-B-W_{X}+B_{X} .
$$

From this and taking into account that for any $v \in V\left(K_{r_{1}, r_{2} \ldots, r_{n}}\right),-1 \leq \#(v) \leq 1$, we obtain that for any part $X$,

$$
-1 \leq W-B-W_{X}+B_{X} \leq 1
$$

By (1) and (2), we have

$$
W-B \geq 1 .
$$

For any part $Y\left(Y \neq X^{\prime}\right)$, by $(2)$, we have

$$
-1 \leq W-B-W_{Y}+B_{Y} \leq 1 .
$$

From this and (3), we obtain that for any part $Y\left(Y \neq X^{\prime}\right)$,

$$
W_{Y}-B_{Y} \geq 0 \text {. }
$$

Let us consider any $v \in X\left(X \neq X^{\prime}\right)$. Clearly,

$$
\#(v)=\sum_{Y, Y \neq X}\left(W_{Y}-B_{Y}\right)=\sum_{Y, Y \neq X, X^{\prime}}\left(W_{Y}-B_{Y}\right)+W_{X^{\prime}}-B_{X^{\prime}}
$$

By $(1)$ and $(4)$, we get $\#(v) \geq 2$, which is a contradiction.

Theorem 1. The graph $K_{r_{1}, r_{2} \ldots, r_{n}}$ has a locally-balanced 2-partition with an open neighborhood if and only if the number of odd parts is even or one.

Proof. Assume that $K_{r_{1}, r_{2} \ldots, r_{n}}$ has $k$ even parts and $s$ odd parts. Suppose that $K_{r_{1}, r_{2} \ldots, r_{n}}$ has a locally-balanced 2-partition with an open neighborhood, but contains odd number $s$ $(s \geq 3)$ odd parts. Lemma 2 implies that for any part $X$ of $K_{r_{1}, r_{2} \ldots, r_{n}},\left|W_{X}-B_{X}\right| \leq 1$. We decompose all the vertices of the graph into three groups as follows:

1. all even parts $X_{i}$ (here, we have $W_{X_{i}}-B_{X_{i}}=0$, by Lemma 2);

2. all odd parts $X_{i}^{\prime}$ with $W_{X_{i}^{\prime}}>B_{X_{i}^{\prime}}$ (here, we have $W_{X_{i}^{\prime}}-B_{X_{i}^{\prime}}=1$, by Lemma 2);

3. all odd parts $X_{i}^{\prime \prime}$ with $W_{X_{i}^{\prime \prime}}<B_{X_{i}^{\prime \prime}}$ (here, we have $W_{X_{i}^{\prime \prime}}-B_{X_{i}^{\prime \prime}}=-1$, by Lemma 2 .).

Let $X_{1}, X_{2}, \ldots, X_{k}$ be parts of the first group; $X_{1}^{\prime}, X_{2}^{\prime}, \ldots, X_{m}^{\prime}$ be parts of the second group; $X_{1}^{\prime \prime}, X_{2}^{\prime \prime}, \ldots, X_{t}^{\prime \prime}$ be parts of the third group. Without loss of generality, we may assume that $m>t$. Consider two cases. 
Case 1: $t=0$.

Clearly, $m \geq 3$. Let us consider a vertex $v \in X_{1}^{\prime}$. By 1 and 2 , we have

$$
\#(v)=\sum_{Y, Y \neq X_{1}^{\prime}}\left(W_{Y}-B_{Y}\right)=\sum_{i=1}^{k}\left(W_{X_{i}}-B_{X_{i}}\right)+\sum_{i=2}^{m}\left(W_{X_{i}^{\prime}}-B_{X_{i}^{\prime}}\right)=m-1 \geq 2,
$$

which is a contradiction.

Case 2: $t>0$.

Let us consider a vertex $v \in X_{1}^{\prime \prime}$. By 1, 2 and 3, we have

$$
\begin{gathered}
\#(v)=\sum_{Y, Y \neq X_{1}^{\prime \prime}}\left(W_{Y}-B_{Y}\right)=\sum_{i=1}^{k}\left(W_{X_{i}}-B_{X_{i}}\right)+\sum_{i=1}^{m}\left(W_{X_{i}^{\prime}}-B_{X_{i}^{\prime}}\right)+ \\
\sum_{i=2}^{t}\left(W_{X_{i}^{\prime \prime}}-B_{X_{i}^{\prime \prime}}\right)=m-(t-1)=(m-t)+1 \geq 2,
\end{gathered}
$$

which is a contradiction.

Now we show that if the number of odd parts is even or one, then $K_{r_{1}, r_{2} \ldots, r_{n}}$ has a locally-balanced 2-partition with an open neighborhood.

First we color even parts uniformly.

Next we consider two cases.

Case A) The number of odd parts is $2 l$. First $l$ odd parts we color as follows: if $X$ is such a part with $2 p+1(p \geq 0)$ vertices, then any $p$ vertices get the color Black and the rest vertices get the color White. Next $l$ odd parts we color similarly by taking the color White instead of Black, and vice versa.

Case B) $X$ is the only odd part with $2 p+1(p \geq 0)$ vertices.

In this case we color any $p$ vertices of $X$ with the color Black and the rest vertices with the color White.

It is easy to see that the above-mentioned 2-partition is a locally-balanced 2-partition of $K_{r_{1}, r_{2} \ldots, r_{n}}$ with an open neighborhood.

Theorem 2. If $m_{1}=0$, then the graph $K_{r_{1}, r_{2} \ldots, r_{n}}$ has a locally-balanced 2-partition with a closed neighborhood if and only if it has no odd part.

Proof. Let $\varphi$ be a locally-balanced 2-partition with a closed neighborhood of $K_{r_{1}, r_{2} \ldots, r_{n}}$. Suppose, to the contrary, that there exists an odd part $X$. Since $m_{1}=0$, we have $|X| \geq 3$. Without loss of generality, we may assume that $W_{X}>B_{X}$. We consider two cases.

Case 1: $B_{X}=0$.

Consider a vertex $v \in X$. Clearly, $\varphi(v)=$ White. This implies that

$$
\#[v]=W-B-W_{X}+B_{X}+1 .
$$

Since $\varphi$ is a locally-balanced 2-partition with a closed neighborhood of $K_{r_{1}, r_{2} \ldots, r_{n}}$, we have

$$
-1 \leq W-B-W_{X}+B_{X}+1 \leq 1
$$

This implies that 


$$
W-B \geq W_{X}-2>0
$$

Case 2: $B_{X}>0$.

Consider a vertex $v \in X$ with $\varphi(v)=$ Black. This implies that

$$
\#[v]=W-B-W_{X}+B_{X}-1 \text {. }
$$

Since $\varphi$ is a locally-balanced 2-partition with a closed neighborhood of $K_{r_{1}, r_{2} \ldots, r_{n}}$, we have

$$
-1 \leq W-B-W_{X}+B_{X}-1 \leq 1
$$

This implies that $W-B \geq W_{X}-B_{X}>0$.

In any case we obtain

$$
W-B>0 .
$$

First we consider the case when for any part $Y, W_{Y} \geq B_{Y}$. Let us consider a vertex $v \in X^{\prime}$ $\left(X^{\prime} \neq X\right)$ with $\varphi(v)=$ White. This implies that

$$
\begin{gathered}
\#[v]=\sum_{Y, Y \neq X^{\prime}}\left(W_{Y}-B_{Y}\right)+1= \\
W_{X}-B_{X}+\sum_{Y, Y \neq X, X^{\prime}}\left(W_{Y}-B_{Y}\right)+1 \geq 2,
\end{gathered}
$$

which is a contradiction.

So, we may assume that there exists a part $X^{\prime}$ such that $W_{X^{\prime}}<B_{X^{\prime}}$.

If $W_{X^{\prime}}=0$, then we consider a vertex $v \in X^{\prime}$. Clearly, $\varphi(v)=$ Black. From this and taking into account that $\varphi$ is a locally-balanced 2-partition with a closed neighborhood of $K_{r_{1}, r_{2} \ldots, r_{n}}$, we have

$$
-1 \leq W-B-W_{X^{\prime}}+B_{X^{\prime}}-1 \leq 1
$$

Hence, $W-B \leq 2-B_{X^{\prime}} \leq 0$, which contradicts (5).

If $W_{X^{\prime}}>0$, then we consider a vertex $v \in X^{\prime}$ with $\varphi(v)=$ White. From this and taking into account that $\varphi$ is a locally-balanced 2-partition with a closed neighborhood of $K_{r_{1}, r_{2} \ldots, r_{n}}$, we have

$$
-1 \leq W-B-W_{X^{\prime}}+B_{X^{\prime}}+1 \leq 1
$$

Hence, $W-B \leq W_{X^{\prime}}-B_{X^{\prime}}<0$, which contradicts (5).

Let $K_{r_{1}, r_{2} \ldots, r_{n}}$ be a complete $n$-partite graph without an odd part. In this case we color each part uniformly.

Lemma 3. If $m_{1}>0$ and $K_{r_{1}, r_{2} \ldots, r_{n}}$ has a locally-balanced 2-partition with a closed neighborhood, then $|W-B| \leq 1$. 
Proof. Let $\varphi$ be a locally-balanced 2-partition with a closed neighborhood of $K_{r_{1}, r_{2} \ldots, r_{n}}$. Consider a vertex $v$ of the part $X$ with $|X|=1$. Since $\varphi$ is a locally-balanced 2-partition with a closed neighborhood of $K_{r_{1}, r_{2} \ldots, r_{n}}$, we have $\#[v]=W-B$, and hence

$$
-1 \leq W-B \leq 1 \text {. }
$$

Remark 1. Clearly, Lemma 3. implies that if $\left|V\left(K_{r_{1}, r_{2} \ldots, r_{n}}\right)\right|$ is even, then $W=B$, and if $\left|V\left(K_{r_{1}, r_{2} \ldots, r_{n}}\right)\right|$ is odd, then $|W-B|= \pm 1$ (in fact, by Lemma 1., we may assume that $W-B=1)$.

Theorem 3. If $m_{1}>0$ and $\left|V\left(K_{r_{1}, r_{2} \ldots, r_{n}}\right)\right|$ is even, then $K_{r_{1}, r_{2} \ldots, r_{n}}$ has a locally-balanced 2-partition with a closed neighborhood if and only if it has no odd part $X$ with at least three vertices.

Proof. Let $\varphi$ be a locally-balanced 2-partition with a closed neighborhood of $K_{r_{1}, r_{2} \ldots, r_{n}}$. Suppose that there exists an odd part $X$ with at least three vertices. We may assume that $W_{X}>B_{X}$. We consider two cases.

Case 1: $B_{X}=0$.

Consider a vertex $v \in X$. Clearly, $\varphi(v)=$ White. This implies that

$$
\#[v]=W-B-W_{X}+B_{X}+1 .
$$

By Remark 1 , we get $\#[v]=1-W_{X} \leq-2$, which is a contradiction.

Case 2: $B_{X}>0$.

Consider a vertex $v \in X$ with $\varphi(v)=$ Black. This implies that

$$
\#[v]=W-B-W_{X}+B_{X}-1
$$

By Remark 1 and taking into account that $W_{X}>B_{X}$, we obtain

$$
\#[v]=B_{X}-W_{X}-1 \leq 2
$$

which is a contradiction.

Now let $K_{r_{1}, r_{2} \ldots, r_{n}}$ be a complete $n$-partite graph with $m_{1}>0$ odd parts. Clearly, $m_{1}$ is even.

Each even part we color uniformly. Then we color $\frac{m_{1}}{2}$ odd parts with the color Black and the other $\frac{m_{1}}{2}$ odd parts with the color White.

It is easy to see that this 2-partition is a locally-balanced 2-partition with a closed neighborhood of $K_{r_{1}, r_{2} \ldots, r_{n}}$.

Theorem 4. If the graph $K_{r_{1}, r_{2} \ldots, r_{n}}$ has an odd number of vertices, $m_{1}>0$ and there exists a part $X$ such that $|X|=2+2 k(k \in \mathbf{N})$, then $K_{r_{1}, r_{2} \ldots, r_{n}}$ has no locally-balanced 2-partition with a closed neighborhood.

Proof. Suppose that $\varphi$ is a locally-balanced 2-partition with a closed neighborhood of $K_{r_{1}, r_{2} \ldots, r_{n}}$. By Remark 1 and Lemma 1, we get

$$
W-B=1 .
$$


Let us consider four cases.

Case 1: $B_{X}=0$.

Let us consider a vertex $v \in X$. Since $\varphi$ is a locally-balanced 2-partition with a closed neighborhood of $K_{r_{1}, r_{2} \ldots, r_{n}}$, we have

$$
\#[v]=W-B-W_{X}+B_{X}+1=2-W_{X} \leq-2,
$$

which is a contradiction.

Case 2: $W_{X}=0$.

Let us consider a vertex $v \in X$. Since $\varphi$ is a locally-balanced 2-partition with a closed neighborhood of $K_{r_{1}, r_{2} \ldots, r_{n}}$, we have

$$
\#[v]=W-B-W_{X}+B_{X}-1=B_{X} \geq 4,
$$

which is a contradiction.

Case 3: $W_{X}>B_{X}>0$.

Let us consider a vertex $v \in X$ with $\varphi(v)=$ Black. Since $\varphi$ is a locally-balanced 2-partition with a closed neighborhood of $K_{r_{1}, r_{2} \ldots, r_{n}}$, we have

$$
\#[v]=W-B-W_{X}+B_{X}-1=B_{X}-W_{X} \leq-2,
$$

which is a contradiction.

Case 4: $0<W_{X} \leq B_{X}$.

Let us consider a vertex $v \in X$ with $\varphi(v)=$ White. Since $\varphi$ is a locally-balanced 2-partition with a closed neighborhood of $K_{r_{1}, r_{2} \ldots, r_{n}}$, we have

$$
\#[v]=W-B-W_{X}+B_{X}+1=2+B_{X}-W_{X} \geq 2,
$$

which is a contradiction.

Theorem 5. If the graph $K_{r_{1}, r_{2} \ldots, r_{n}}$ has an odd number of vertices, $m_{1}>0$ and each part $X$ of the graph has either two vertices or an odd number of vertices, then $K_{r_{1}, r_{2} \ldots, r_{n}}$ has a locally-balanced 2-partition with a closed neighborhood if and only if $m_{1} \geq 2 m_{2}+m_{\geq 3}-1$.

Proof. Suppose that $\varphi$ is a locally-balanced 2-partition with a closed neighborhood of $K_{r_{1}, r_{2} \ldots, r_{n}}$. By Remark 1 and Lemma 1, we have

$$
W-B=1 .
$$

Let us consider a part $X$ with only two vertices $u$ and $v$.

If $\varphi(v)=$ White and $\varphi(u)=$ Black, then since $\varphi$ is a locally-balanced 2-partition with a closed neighborhood of $K_{r_{1}, r_{2} \ldots, r_{n}}$, we have

$$
\#[v]=W-B-W_{X}+B_{X}+1=2,
$$

a contradiction.

Similarly, if $\varphi(v)=$ Black and $\varphi(u)=$ White, then we consider the vertex $u$.

If $\varphi(v)=\varphi(u)=$ Black, then since $\varphi$ is a locally-balanced 2-partition with a closed neighborhood of $K_{r_{1}, r_{2} \ldots, r_{n}}$, we have

$$
\#[v]=W-B-W_{X}+B_{X}-1=2,
$$


a contradiction.

This implies that $\varphi(v)=\varphi(u)=$ White.

Let $X$ be an odd part with at least three vertices.

If $W_{X}=0$, then by considering a vertex $v \in X$ and taking into account that $\varphi$ is a locally-balanced 2-partition with a closed neighborhood of $K_{r_{1}, r_{2} \ldots, r_{n}}$, we obtain

$$
\#[v]=W-B-W_{X}+B_{X}-1=B_{X} \geq 3,
$$

a contradiction.

If $0<W_{X}<B_{X}$, then by considering a vertex $v \in X$ with $\varphi(v)=$ White and taking into account that $\varphi$ is a locally-balanced 2-partition with a closed neighborhood of $K_{r_{1}, r_{2} \ldots, r_{n}}$, we obtain

$$
\#[v]=W-B-W_{X}+B_{X}+1=2+B_{X}-W_{X} \geq 3,
$$

a contradiction. This shows that

$$
W_{X}>B_{X}
$$

Let us consider two cases.

Case 1: $|X|=3$.

By (7), we have that there are two possible cases: all vertices of $X$ are White or two of them are White and the last one is Black.

If $W_{X}=3$ and $B_{X}=0$, then for any $v \in X$, we have

$$
\#[v]=W-B-W_{X}+B_{X}+1=-1 .
$$

This implies that for any $v \in X,-1 \leq \#[v] \leq 1$.

If $W_{X}=2$ and $B_{X}=1$, then for any $v \in X$, we have either $\#[v]=W-B-W_{X}+B_{X}+1=$ 1 (if $\varphi(v)=$ White) or $\#[v]=W-B-W_{X}+B_{X}-1=-1$ (if $\varphi(v)=$ Black).

This implies that for any $v \in X,-1 \leq \#[v] \leq 1$.

Case 2: $|X| \geq 5$.

By (7), we have $W_{X}-B_{X} \geq 1$.

Let us show that $W_{X}-B_{X}=1$.

Suppose, to the contrary, that $W_{X}-B_{X} \geq 3$.

If $B_{X}=0$, then for any $v \in X$, we have

$$
\#[v]=W-B-W_{X}+B_{X}+1 \leq-3,
$$

which is a contradiction.

If $W_{X}>B_{X}>0$. For any $v \in X$ with $\varphi(v)=$ Black, we have

$$
\#[v]=W-B-W_{X}+B_{X}-1 \leq-3,
$$

which is a contradiction.

This shows that $W_{X}-B_{X}=1$.

So, we obtain that if $X$ is a part with at least two vertices, then we have the following three possible cases:

a) If $X$ is an odd part with three vertices, then

$$
\text { either } W_{X}-B_{X}=1 \quad \text { or } \quad W_{X}-B_{X}=3 \text {; }
$$


b) If $X$ is an odd part with at least five vertices, then

$$
W_{X}-B_{X}=1
$$

c) If $X$ has two vertices, then

$$
W_{X}-B_{X}=2 \text {. }
$$

By (6), (8),(9),(10), we get

$$
\begin{gathered}
m_{1}=\sum_{X,|X|=1}\left(B_{X}+W_{X}\right) \geq \sum_{X,|X|=1}\left(B_{X}-W_{X}\right)=B-W+\sum_{X,|X|=2}\left(W_{X}-B_{X}\right)+ \\
\sum_{X, W_{X}-B_{X}=1}\left(W_{X}-B_{X}\right)+\sum_{X, W_{X}-B_{X}=3}\left(W_{X}-B_{X}\right)=B-W+2 \mid\{X: X \text { is a part with }|X|=2\} \mid+
\end{gathered}
$$

$\mid\left\{X: X\right.$ is a part with $\left.W_{X}-B_{X}=1\right\}|+3|\left\{X: X\right.$ is a part with $\left.W_{X}-B_{X}=3\right\} \mid \geq$

$B-W+2 \mid\{X: X$ is a part with $|X|=2\}|+|\left\{X: X\right.$ is a part with $\left.W_{X}-B_{X}=1\right\} \mid+$

$\mid\left\{X: X\right.$ is a part with $\left.W_{X}-B_{X}=3\right\}|=B-W+2|\{X: X$ is a part with $|X|=2\} \mid+$

$\mid\{X: X$ is an odd part with at least three vertices $\} \mid=-1+2 m_{2}+m_{\geq 3}$.

Now let $m_{1} \geq 2 m_{2}+m_{\geq 3}-1$. We construct a 2-partition of $K_{r_{1}, r_{2} \ldots, r_{n}}$ as follows:

1) Each vertex $v \in X(|X|=2)$, we color with the color White;

2) For any odd part $X$ with $|X|=2 p+1(p \in \mathbf{N})$ vertices, we color $p+1$ vertices of $X$ with the color White, and the rest $p$ vertices we color with the color Black;

3) We take any $\left(2 m_{2}+m_{\geq 3}-1\right)$ parts with only one vertex of the graph and we color each vertex in these parts with the color Black. Since $\left|V\left(K_{r_{1}, r_{2} \ldots, r_{n}}\right)\right|$ is odd and $m_{1} \geq$ $2 m_{2}+m_{\geq 3}-1$, we have that $m_{1}-\left(2 m_{2}+m_{\geq 3}-1\right)$ is even and non-negative. The vertices of the remaining $m_{1}-\left(2 m_{2}+m_{\geq 3}-1\right)$ parts we color uniformly.

It is not difficult to see that this 2-partition is a locally-balanced 2-partition with a closed neighborhood of $K_{r_{1}, r_{2} \ldots, r_{n}}$. 


\section{References}

[1] C. Berge, Graphs and Hypergraphs, Elsevier Science Ltd, 1985.

[2] S.V. Balikyan, "On existence of certain locally-balanced 2-partition of a tree", Mathematical Problems of Computer Science, Vol. 30, pp. 25-30, 2008.

[3] S.V. Balikyan and R. R. Kamalian, "On existence of 2-partition of a tree, which obeys the given priority", Mathematical Problems of Computer Science, Vol. 30, pp. 31-35, 2008.

[4] S.V. Balikyan and R. R. Kamalian, "Necessary and sufficient condition for existence of locally-balanced 2-partition of a tree under the extended definition of a neighbourhood of a vertex", Mathematical Problems of Computer Science, vol. 31, pp. 116-121, 2008.

[5] A. H. Gharibyan and P.A. Petrosyan, "Locally-balanced 2-partitions of complete multipartite graphs", 6th Polish Combinatorial Conference, Bedlewo, Poland, p. 19, 2016.

[6] A.H. Gharibyan and P.A. Petrosyan, "On locally-balanced 2-partitions of some graphs", Proceedings of the CSIT Conference, Yerevan, pp. 196-197, 2017.

[7] A. Hajnal and E. Szemeredi, "Proof of a conjecture of P. Erdös", Combinatorial theory and its applications, II (Proc. Colloq., Balatonfred, 1969), North-Holland, pp. 601-623, 1970.

[8] G. Chartrand and P. Zhang, Chromatic Graph Theory, Discrete Mathematics and Its Applications, CRC Press, 2009.

[9] W. Meyer, "Equitable coloring", American Mathematical Monthly, vol. 80, no. 8, pp. 920-922, 1973.

[10] A.V. Kostochka, "Equitable colorings of outerplanar graphs", Discrete Mathematics vol. 258, pp. 373-377, 2002.

[11] J. Kratochvil, "Complexity of hypergraph coloring and Seidel's switching", Graph Theoretic Concepts in Computer Science, 29th International Workshop, WG 2003, Elspeet, The Netherlands, Revised Papers, vol. 2880, pp. 297-308, June 19-21, 2003.

[12] S. V. Balikyan and R. R. Kamalian, "On NP-completeness of the problem of existence of locally-balanced 2-partition for bipartite graphs $G$ with $\Delta(G)=3$ ", Doklady NAN $R A$, vol. 105, no. 1, pp. 21-27, 2005.

[13] S.V. Balikyan and R.R. Kamalian, "On NP-completeness of the problem of existence of locally-balanced 2-partition for bipartite graphs $G$ with $\Delta(G)=4$ under the extended definition of the neighbourhood of a vertex", Doklady $N A N R A$, vol. 106, no. 3, pp. 218-226, 2006.

[14] D. de Werra, "On good and equitable colorings", In Cahiers du C.E.R.O., vol. 17, pp. 417-426, 1975.

[15] D.B. West, Introduction to Graph Theory, Prentice-Hall, New Jersey, 2001.

\section{Submitted 02.10.2017, accepted 26.01.2018.}




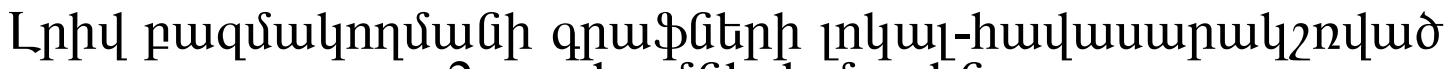 2-unpnhnuditiph ưuph
}

U. RuphpjuG i T. ThinnnujuG

\section{Uরuนnnนnน์}

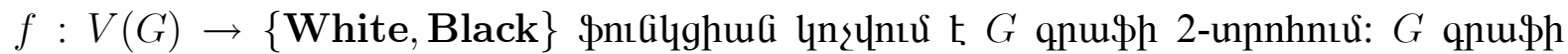

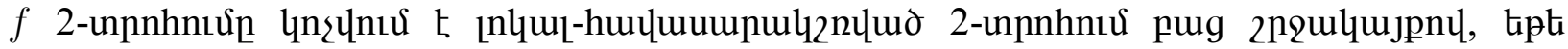
quर्umuluma $v \in V(G)$ ququph huर्um untinh nıGh

$$
\|\left\{u \in N_{G}(v): f(u)=\text { White }\right\}|-|\left\{u \in N_{G}(v): f(u)=\text { Black }\right\} \| \leq 1,
$$

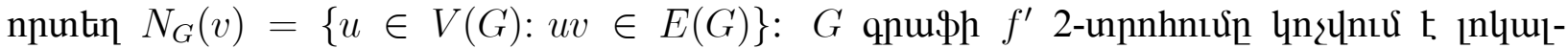

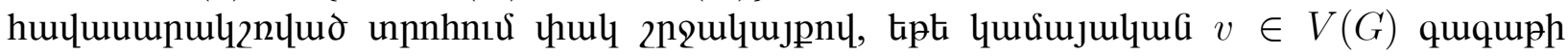
huviup untinh nıGip

$$
\|\left\{u \in N_{G}[v]: f^{\prime}(u)=\text { White }\right\}|-|\left\{u \in N_{G}[v]: f^{\prime}(u)=\text { Black }\right\} \| \leq 1,
$$

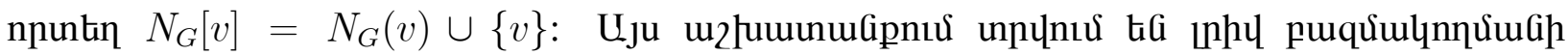

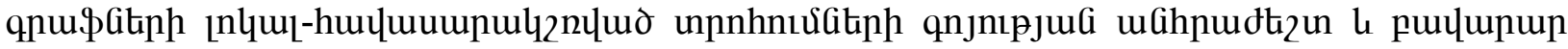
uщujuiugitip:

\section{О локально-сбалансированных 2-разбиениях полных многодольных графов}

\author{
А. Гарибян и П. Петросян
}

\section{Аннотация}

2-разбиением графра $G$ называется фуннцция $f: V(G) \rightarrow\{$ White, Black\}. 2-разбиение $f$ графра $G$ называется локально-сбалансированным с открытой окрестностью, если для любой вершины $v \in V(G)$

$$
\|\left\{u \in N_{G}(v): f(u)=\text { White }\right\}|-|\left\{u \in N_{G}(v): f(u)=\text { Black }\right\} \| \leq 1,
$$

где $N_{G}(v)=\{u \in V(G): u v \in E(G)\}$. 2-разбиение $f^{\prime}$ графа $G$ называется локальносбалансированным с закрытой окрестностью, если для любой вершины $v \in V(G)$

$$
\|\left\{u \in N_{G}[v]: f^{\prime}(u)=\text { White }\right\}|-|\left\{u \in N_{G}[v]: f^{\prime}(u)=\text { Black }\right\} \| \leq 1,
$$

где $N_{G}[v]=N_{G}(v) \cup\{v\}$. В настоящей работе даются необходимые и достаточные условия существования локально-сбалансированных 2-разбиений полных многодольных графов. 